\title{
Development of an ultrasound training curriculum in a limited resource international setting: successes and challenges of ultrasound training in rural Rwanda
}

\author{
Sachita Shah • Vicki E. Noble • Irenee Umulisa • \\ J. M. V. Dushimiyimana • Gene Bukhman • \\ Joia Mukherjee $\cdot$ Michael Rich $\cdot$ Henry Epino
}

Received: 29 June 2008 / Accepted: 20 July 2008 / Published online: 25 September 2008

(C) Springer-Verlag London Ltd 2008

\begin{abstract}
Background Over the last decade, the diffusion of ultrasound technology to nontraditional users has been rapid and farreaching. Much research and effort has been focused on developing an ultrasound curriculum and training and practice guidelines for these users. The potential for this diagnostic tool is not limited to the developed world and in many respects ultrasound is adaptable to limited resource international settings. However, needs-based curriculum development, training guidelines, impact on resource utilization, and
\end{abstract}

S. Shah $(\bowtie) \cdot$ V. E. Noble $\cdot$ H. Epino

Department of Emergency Medicine, Zero Emerson \#3B,

Massachusetts General Hospital,

55 Fruit St.,

Boston, MA, USA

e-mail: Sachita.shah@gmail.com

I. Umulisa

Kirehe Hospital \& Rwinkwavu Hospital,

Partners in Health/Inshuti Mu Buzima (PIH/IMB),

Rwinkwavu, Rwanda

J. M. V. Dushimiyimana

Kirehe Hospital \& Rwinkwavu Hospital,

Partners in Health/Inshuti Mu Buzima (PIH/IMB),

Kirehe, Rwanda

G. Bukhman · J. Mukherjee $\cdot$ M. Rich

Division of Social Medicine and Health Inequalities,

Brigham and Women's Hospital,

75 Francis St.,

Boston, MA, USA

G. Bukhman $\cdot$ J. Mukherjee $\cdot$ M. Rich

Program in Infectious Disease and Social Change,

Department of Social Medicine, Harvard Medical School,

Boston, MA, USA sustainability are not well studied in the developing world setting.

Aims We review one method of introducing applicable curriculum, training local providers, and sustaining a comprehensive ultrasound program.

Methods Two rural Rwandan hospitals affiliated with a US nongovernmental organization participated in a pilot ultrasound training program. Prior to introduction of ultrasound, local physicians completed a survey to determine the perceived importance of various ultrasound scan types. Hospital records were also reviewed to determine disease and presenting complaint prevalence as part of an initial needs assessment and to define our curriculum. We hypothesized certain studies would be more utilized and have a greater impact given available treatment resources.

Results We review here the choice of curriculum, the training plan, helpful equipment specifications, and implementation of ongoing measures of quality assessment and sustainability. Our 9-week lecture and practice-based ultrasound curriculum included obstetrics, abdominal, renal, hepatobiliary, cardiac, pleural, vascular, and procedural ultrasound.

Conclusions While ultrasound as a diagnostic modality for resource-poor parts of the world has generated interest for years, recent advances in technology have brought ultrasound again to the forefront as a sustainable and high impact technology for resource-poor developing world nations. From our experience in rural Rwanda, we conclude that ultrasound remains helpful in patient care and the diagnostic impact is enhanced by choosing the correct applications to implement. We also conclude that ultrasound is a teachable skill, with a several week intensive training period involving hands-on practice skills and plans for long-term learning and have begun a second phase of evaluating knowledge retention for this introductory program. 
Keywords International ultrasound · Ultrasound curriculum · Ultrasound training $\cdot$ Imaging technology

\section{Introduction}

Lack of adequate health care facilities, personnel, and diagnostic tools remains a major barrier to health care delivery in rural Sub-Saharan Africa and contributes to the major causes of death in this region: acquired immunodeficiency syndrome (AIDS), malaria, tuberculosis, malnutrition, and obstetric complications [1]. Nongovernmental organizations are focusing attention on the introduction of appropriate, cost-effective technology to improve clinical outcomes. Many hypothesize that an imaging modality such as ultrasound could help boost the diagnostic capacity of rural health care centers and thereby ameliorate care delivery by improving diagnostic accuracy to better use scarce resources [2-4]. The impact of this technology diffusion has never been quantified, and questions remain regarding best methods for teaching ultrasound in these settings, which ultrasound applications are appropriate $[5,6]$, and how ultrasound diagnostic capability will impact patient care, outcomes, and resource utilization. This study reviews one approach for initiating a diagnostic ultrasound program in a resource-poor setting. We will discuss issues of curriculum, training, and quality assurance that arose during the first phases of ultrasound introduction in two rural Rwandan hospitals.

\section{Methods}

Our study setting consisted of two rural hospitals located in the villages of Kirehe and Rwinkwavu in Eastern Province, Rwanda. These hospitals are affiliated with Partners in Health, a US-based nongovernmental organization that provides financial, educational, and health care staff support to a population of over 600,000 people.

Prior to the introduction of ultrasound training, we performed a needs assessment. The Rwandese physicians completed a survey regarding prior ultrasound experience, and hospital records were reviewed to determine common presenting complaints and typical patient demographics. This information was synthesized with local referral capacity for surgeons and specialists, inhospital treatment options, and existing diagnostic capacity to determine which ultrasound applications would likely have the most impact on patient outcome and care.

Ultrasound trainings were conducted by a fourth year emergency medicine resident with several months of prior ultrasound training during residency (including obstetric ultrasound) and credentialing certification as outlined by the American College of Emergency Physicians with one exception, the cardiac echo lecture was given by a visiting cardiologist. All local staff physicians were invited to attend the trainings; 10 of the 15 completed the entire training.

This study was approved by the sponsoring hospital's Institutional Review Board and the Rwandan Ethics Committee.

\section{Findings}

Needs assessment

Initial needs assessments at both hospitals were conducted via a survey of 15 staff physicians and hospital record review.

In response to survey questions about prior ultrasound experience, all physicians indicated $0-4 \mathrm{~h}$ of prior ultrasound training, solely in obstetric ultrasound. In ranking various ultrasound applications, all 15 trainees rated obstetrics most important. Focus group discussion revealed that barriers to obtaining ultrasound services included distance, time, and cost for transfers, concern over lack of monitoring during transfers, and ultrasound charges. They also expressed dissatisfaction with the quality of ultrasound reports and the inability to view images in addition to written reports.

All 2007 hospital and prenatal clinic logbooks were reviewed to determine patient demographics, admitting and discharge diagnoses, reasons for patient transfers to outside hospitals, and causes of death. Record review suggested that the majority of presentations were related to malnutrition, infectious diseases, or obstetrics. We noted many cases where ultrasound could be potentially useful such as patients with rheumatic heart disease, congestive heart failure, tuberculosis (TB)-related pericardial and pleural effusions, TB- or cirrhosis-related ascites, and chronic kidney disease. In 2007, the annual census of admitted patients per month at Kirehe Hospital was 222 (range: 142-322) with approximately 40\% pediatric admissions. Each hospital averaged 109 (range: 42149) live births per month in 2007, with cesarean section capacity only available at Rwinkwavu Hospital. At both hospitals diagnostic imaging prior to ultrasound was limited to hand-developed radiographs (approximately 10-25 radiographs per day at each hospital). Regionally, supplementary diagnostic imaging (computed tomography scanning) and specialty surgical, obstetric, cardiology, and neurosurgical consultation was available via ambulance transfer but required several hours transfer time over rough terrain.

\section{Curriculum}

The ultrasound training curriculum replicated at both of the Rwandan hospitals spanned 9 weeks and included lectures followed by practical hands-on sessions. The first overview 
lecture covered principles of ultrasound physics, machine specifics, potential uses of ultrasound, the plan for the lecture series and training, and image and data sheet recording. The next lecture in the series covered obstetric ultrasound including first trimester scanning, ectopic and molar pregnancies, estimating gestational age, and evaluation of fetal position, cervix, and placenta. The cardiac echo lecture covered evaluation for mitral stenosis, estimation of global left ventricular function, and pericardial effusion. Further lectures included hepatobiliary ultrasound, including evaluation for amebic abscess, echinococcal cysts, and cholecystitis, as well as renal ultrasound for evaluation of chronic kidney disease including human immunodeficiency virus (HIV) nephropathy. Advanced applications discussed were deep venous thrombosis (DVT) evaluation, vascular access, abscess evaluation, and procedural guidance using ultrasound.

All participants were given copies of the presentations and two ultrasound review books were donated to the hospital libraries. After completion of the lecture series, bimonthly image review sessions were initiated.

\section{Training plan}

At both Kirehe and Rwinkwavu Hospitals, 1-h lectures were conducted three times per week for the first 3 weeks at each site, and then once per week for the remaining time. Each lecture was followed by $1-2 \mathrm{~h}$ of scanning practice.

In addition to focused training times, the ultrasound instructor joined the local staff physicians on ward rounds daily during the 9-week period and encouraged utilization of the ultrasound in routine clinical practice and provided immediate feedback on ultrasound exams performed by the local staff. These proctored exams provided valuable one-on-one training to improve scanning techniques. As positive findings emerged during routine ultrasound exams, the instructor provided additional clinical teaching to modify patient care plans as needed based on ultrasound results.

Sustainability and quality assurance

At each hospital, a local staff physician who showed particular interest as well as proficiency with the ultrasound was identified to become the "ultrasound coordinator" at each site. These physicians were entrusted with care of the ultrasound machine, gel and supply ordering, ultrasound logbook upkeep, and were taught how upload images from the ultrasound machine to their personal computer. Through the ultrasound coordinator, physicians were able to send images via e-mail for ongoing quality assurance after the training period ended. Physicians regularly received feedback via e-mail regarding scans they had completed after review by the trainer, which allowed them to feel supported and provided ongoing evidence that ultrasound continued to be useful even after the initial training period ended.

\section{Machine specifics}

We used two SonoSite MicroMaxx ultrasounds for this training curriculum; however, other machines may be well suited for this environment with regards to the following key interests. Of utmost importance is machine durability resistance to heat, water, and dust is necessary for any machine that will have longevity in a rustic environment. As many of the hospital wards were in separate buildings or clinics and some patients required home visits for ultrasound diagnostics, portability, i.e, a machine that can easily be hand carried over rough terrain, ensures that the machine will be able to be used in all relevant clinical environments. As electricity may be intermittent, a battery-operated machine with short recharge time is ideal, and a voltage stabilizer should be used for safety of the machine. A machine that can provide high quality two-dimensional images without sacrificing other features is required. Regarding probe choice, we used a curvilinear 3-5 MHz (abdominal) probe, an endocavitary probe, and a microconvex lowfrequency 5-8 $\mathrm{MHz}$ probe for vascular, obstetric, pediatric, and cardiac applications and found these sufficient to meet all needs. Lastly, the machine should be accompanied by a storage bag with room for gel, towels, probe covers, and cleaning supplies.

\section{Conclusions}

From our experience in rural Rwanda, we conclude that ultrasound remains helpful in patient care and the diagnostic impact is enhanced by choosing the correct applications to implement. We also conclude that ultrasound is a teachable skill, with a several week intensive training period involving hands-on practice skills and plans for long-term learning and have begun a second phase of evaluating knowledge retention for this introductory program. Essential to any successful ultrasound program is the identification of a local ultrasound coordinator to continue the enthusiasm and program development after the initial training period. In addition, it is helpful to make available ongoing quality assurance and image feedback via e-mail or web-based teaching tools. We did not anticipate but were delighted to find that professional satisfaction of local health providers may be increased by the training of a new skill such as ultrasound and may begin to spark an interest in academic medicine in physician trainees in the developing world. 
Future research is needed to gauge diagnostic accuracy of ultrasound and impact on patient outcomes and resource utilization in this type of setting.

\section{References}

1. The world health report 1996-fighting disease, fostering development. Report of the Director-General. World Health Organization, Geneva

2. Hoyer P, Weber M (1997) Ultrasound in developing world. Lancet 350:1330

3. Mindel S (1997) Role of imager in developing world. Lancet 350:426-429

4. Mets T (1991) Clinical ultrasound in developing countries. Lancet 337:358

5. Tshibwabwa ET, Mwaba P, Bogle-Taylor J et al (2000) Four-year study of abdominal ultrasound in 900 Central African adults with AIDS referred for diagnostic imaging. Abdom Imaging 25:290-296

6. Steinmetz JP, Berger JP (1999) Ultrasonography as an aid to diagnosis and treatment in a rural African hospital: a prospective study of 1,119 cases. Am J Trop Med Hyg 60(1):119-123

Sachita Shah received her medical degree from the University of Washington, Seattle, WA in 2004 and completed her residency training at the Harvard Affiliated Emergency Medicine Residency at Massachusetts General Hospital and Brigham \& Women's Hospital, Boston, MA. She is currently a clinical fellow in emergency ultrasound at Highland Hospital, Oakland, CA. Her research interests focus on the impact of ultrasound in the developing world.

Vicki Noble is Director of Emergency Ultrasound at Massachusetts General Hospital, where she is an attending physician in Emergency Medicine. She currently serves as faculty mentor for Harvard residents interested in ultrasound.

Irenee Umulisa completed her medical training at Butare Medical School in Rwanda in 2006 and now serves as a staff physician at Rwinkwavu Hospital, a Partners in Health affiliated site in rural Rwanda. She has been appointed the Ultrasound Coordinator at PIH Rwinkwavu Hospital.
JMV Dushimiyimana completed his medical training at Butare Medical School in 2007, and began work for Partners in Health in Kirehe Hospital, Rwanda later that year. $\mathrm{He}$ is the Ultrasound Coordinator and Director of Women's and Maternal Health at Kirehe Hospital.

Gene Bukhman is a cardiologist and medical anthropologist in the Department of Social Medicine at Harvard Medical School and the Division of Social Medicine and Health Inequalities at the Brigham and Women's Hospital, Boston, MA. An expert in cardiovascular ultrasound, he focuses on the development and evaluation of heart failure services in resource-poor settings.

Joia Mukherjee is an Assistant Professor in the Division of Social Medicine and Health Inequalities at the Brigham \& Women's Hospital, Boston, MA and since 2000, she has served as the Medical Director of Partners In Health. Dr. Mukherjee consults for the World Health Organization on the treatment of HIV and MDR-TB and is an author of both adult and pediatric WHO guidelines for the management of HIV in resource-limited settings.

Michael Rich is an instructor in the Division of Social Medicine and Health Inequalities at the Brigham \& Women's Hospital, Boston, MA and joined Partners in Health in 2000 and has served as the PIH Country Director for Rwanda since 2005. He is the lead author on the WHO guidelines for the management of MDR-TB and a member of the World Health Organization's Green Light Committee of DOTS-Plus for MDR-TB.

Henry Epino is the Medical Director of Partners in Health programs in Rwanda since 2005 and an emergency medicine physician at Massachusetts General Hospital. He has worked clinically for PIH since 1997 and serves as a mentor for many residents interested in international humanitarian work. 